\title{
Testosterone Improved Hyperalgesia in a Patient Under Methadone Maintenance Treatment
}

\author{
Nader Charkhgard ${ }^{1,}$, , Shahram Naderi ${ }^{1}$, Kamyar Ghani $^{1}$ and Mahboubeh Manouchehrabadi ${ }^{1}$ \\ ${ }^{1}$ Department of Neuroscience and Addiction Studies, School of Advanced Technologies in Medicine, Tehran University of Medical Sciences, Tehran, Iran \\ "Corresponding author: Department of Neuroscience and Addiction Studies, School of Advanced Technologies in Medicine, Tehran University of Medical Sciences, Tehran, Iran. \\ Email: ncharkhgard@razi.tums.ac.ir
}

Received 2021 November 22; Revised 2022 January 18; Accepted 2022 January 24.

\begin{abstract}
Introduction: Long-term use of narcotics causes hyperalgesia in some patients with an unknown mechanism known as opioidinduced hyperalgesia $(\mathrm{OIH})$.

Case Presentation: Testosterone was prescribed to a 43-year-old man suffering from the effects of OIH syndrome due to long-term use of methadone. Testosterone prescription significantly reduced the hyperalgesia.

Conclusions: Testosterone may have a therapeutic value in $\mathrm{OIH}$.
\end{abstract}

Keywords: Opioid, Hyperalgesia, Testosterone

\section{Introduction}

Opioids are a category of natural and synthetic products mostly used for ache management and opioid dependency (1). They are powerful analgesics widely prescribed for the management of acute and chronic pain, and their use has quickly grown in current years (2). Opioids exert their analgesic outcomes through binding to opioid receptors in the brain and spinal twine, and are coupled with Gproteins. This leads to the block of calcium and potassium channels, which in turn decreases the neuronal excitability $(1,3)$. Opiates potently suppress the hypothalamicpituitary-gonadal axis (2).

Testosterone plays a vital role in the development of male reproductive organs and other sexual traits such as the growth of body hair, bone, muscle mass $(3,4)$, and body composition $(4,5)$. It also changes the behavior and life style. Decreased levels of testosterone can result in reduced libido, fatigue, reduced muscles mass, osteopenia $(5,6)$, diabetes, osteoporosis, and bone loss $(3,4)$. This syndrome is often called opioid-induced androgen deficiency (OPIAD) $(5,6)$. Testosterone treatment in men with hypogonadism has a therapeutic effect on many male disorders (6). Another symptom of patients with substance use disorders is hyperalgesia due to the drug abuse and the factors affecting it (7). Male hypogonadism refers to a condition in which the body is unable to produce enough testosterone $(8,9)$. Various conditions, such as taking certain medications and drugs, can reduce testosterone levels in men (10).

Is pain a unique sensory experience that reflects each person's perception of the threat to their health? One of the factors influencing the tendency to use drugs, especially narcotics, is pain $(9,10)$, and in particular chronic pain. Chronic pain is also very common in people receiving opioid agonists. This chronic pain during maintenance therapy with agonists is one of the reasons for increasing the dose of agonists (methadone), which paradoxically causes progressive pain in patients (11).

Opioid-induced hyperalgesia $(\mathrm{OIH})$ may be a development whereby opioids increase patients' pain sensitivity, complicating their use in physiological condition $(7,10)$. $\mathrm{OIH}$ is a paradoxical reaction in which the patient takes drugs to treat pain but actually becomes more sensitive to painful stimuli (11). Although very little is known about the prevalence of OIH (11), it seems to be high among opioid patients (12). cold pressor test (CPT) is a well-known test to help identify hyperalgesic patients (12). In this study, CPT was used to diagnose and follow-up hyperalgesia in patients. To do the test, a plastic cooler was filled with ice packs and nearly two-thirds of water. We added crushed ice to the water to keep the temperature around $2^{\circ} \mathrm{C}$, so that the temperature does not change much when recording the pain. During the test, the patient was in sitting position. He was requested to dip one hand into the water (up to $1 \mathrm{~cm}$ above the wrist), keep the hand open, and not to 
touch any of the ice packs. The amount of time that the patient can tolerate this situation determines his sensitivity to pain. This test is used to determine the pain sensitivity threshold (pain onset time) and pain tolerance (maximum time the person tolerates) $(13,14)$.

Possible mechanisms concerned in $\mathrm{OIH}$ include involvement of central sensitization with glutaminergic activation and genetic mechanisms. The two most widely used methods for treating these patients are N-methyl-Daspartate (NMDA) antagonists and opioid rotation. However, the diagnostic criteria have not been well defined yet (11).

\section{Case Presentation}

Our case was a 43-year-old man holding a high school diploma with an average height of $171 \mathrm{~cm}$ and weight of 85 $\mathrm{kg}$. His personal hygiene was good. He was maintaining good eye contact. His sitting posture was much relaxed. The patient was quite motivated to seek treatment.

The patient had a history of using drugs for 20 years (Figure 1). He started smoking cigarettes and using cannabis at the age of 19, and stopped using cannabis two years later. Since he was about 20 years old, he sometimes took prescription drugs (acetaminophen codeine) due to pain in the knees. He started using opium at the age of 23. Also, he tried to quit using drugs for six times (detoxification without drug methods and using buprenorphine); the maximum period of not using drugs was three months. Until five years ago, he used opium and opium juice; then methadone maintenance treatment was started for him. According to his self-declaration and clinical tests twice a month, he had not used opium and its derivatives and other drugs in the last three years. There was no history of substance abuse in the patient's family. Also, in the workplace, two ill employees used drugs.

Most of the routine tests (CBC diff \& LFT) of the patient were normal except for the reduction of free and total serum testosterone levels. Then, once a week, 100 mg of testosterone was injected intramuscularly, and this treatment was continued for three months. During this period, a monthly CPT was performed, but the amount of methadone use was not changed. The patient's testosterone level was measured three months later (Figure 2).

The patient's sexual desire improved, he had more energy to do daily tasks, and he felt less tired. Hot flashes occurred only once in the last three months. The patient's abdominal circumference decreased to $102 \mathrm{~cm}$ (he was on a diet as well), and his testosterone level increased (Figure 2). Without reducing the amount of methadone consumed by the patient during this period, from the first month onwards, the time of feeling the pain and the patient's max- imum ability to hold his hand in iced water gradually increased (Figure 3).

\section{Discussion}

According to some studies, the prevalence of $\mathrm{OIH}$ is related to gender, and somehow it is associated with fibromyalgia. Fibromyalgia is more common in women, and one of its new treatments is testosterone. There is evidence that low testosterone levels are associated with pain $(12,13)$, and taking testosterone supplements can improve the patient's condition and pain perception. In our case, the symptoms were controlled and improved by taking testosterone and not reducing the dose of narcotics.

At the beginning of the study, the patient's complaints included hyperalgesia, decreased libido, failure to orgasm, decreased energy, difficulty concentrating, hot flashes, and irritability. Decreased testosterone levels may have been a factor in the patient's condition during treatment, which was largely due to hypogonadism (except for the patient's concentration and irritability), especially hyperalgesia. To the best of our knowledge, no other intervention has been performed to cure such patients' hyperalgesia. However, it is likely that the decrease in the patient's testosterone levels might be the factor affecting the OIH process.

\subsection{Conclusions}

Some studies have shown that OIH is not related to the dose of the narcotics and may result from any dose of opioid. With each dose of opioid, OIH is formed; however, respondents normally managed OIH by opioid dose reduction, administering a nonopioid adjuvant, or discontinuing opioids (10). Contrary to the evidence showing a link between testosterone levels and $\mathrm{OIH}$ in patients, undergoing maintenance therapy with agonists should be further studied. Given that one of the reasons for the unintended increase in the dose of agonist drugs is to treat hyperalgesia (14), this case report showed that increasing the dose of methadone can reduce the level of testosterone in the blood, and thus increase hyperalgesia in the patient. Different hypotheses about hyperalgesia caused by narcotics have not led to a proper solution and treatment for these patients. In addition, in many patients with $\mathrm{OIH}$, there is still hyperalgesia even after stopping agonist treatment, which might be due to the inherently low blood testosterone levels; this pain can also lead to the relapse of drugs. However, drug use leads to a further decrease in testosterone in the patient and increases hyperalgesia. This means that OIH occurs in patients with lower testosterone levels, and increased serum testosterone levels can help treat and improve the patients' OIH. Further studies are needed to link serum testosterone levels to OIH. 


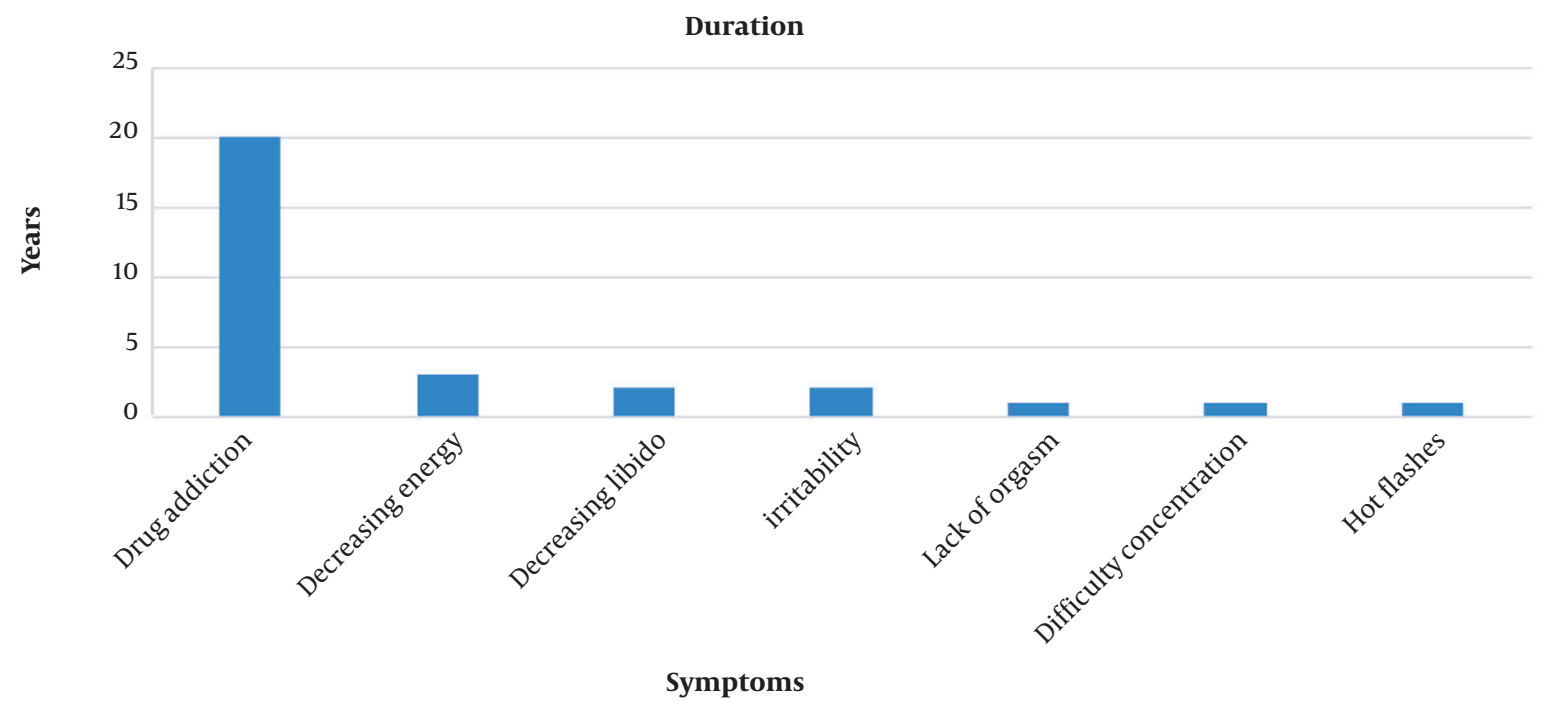

Figure 1. Presenting complaints and duration of the symptoms

Testosterone Level before and 3 month after Starting Tretment

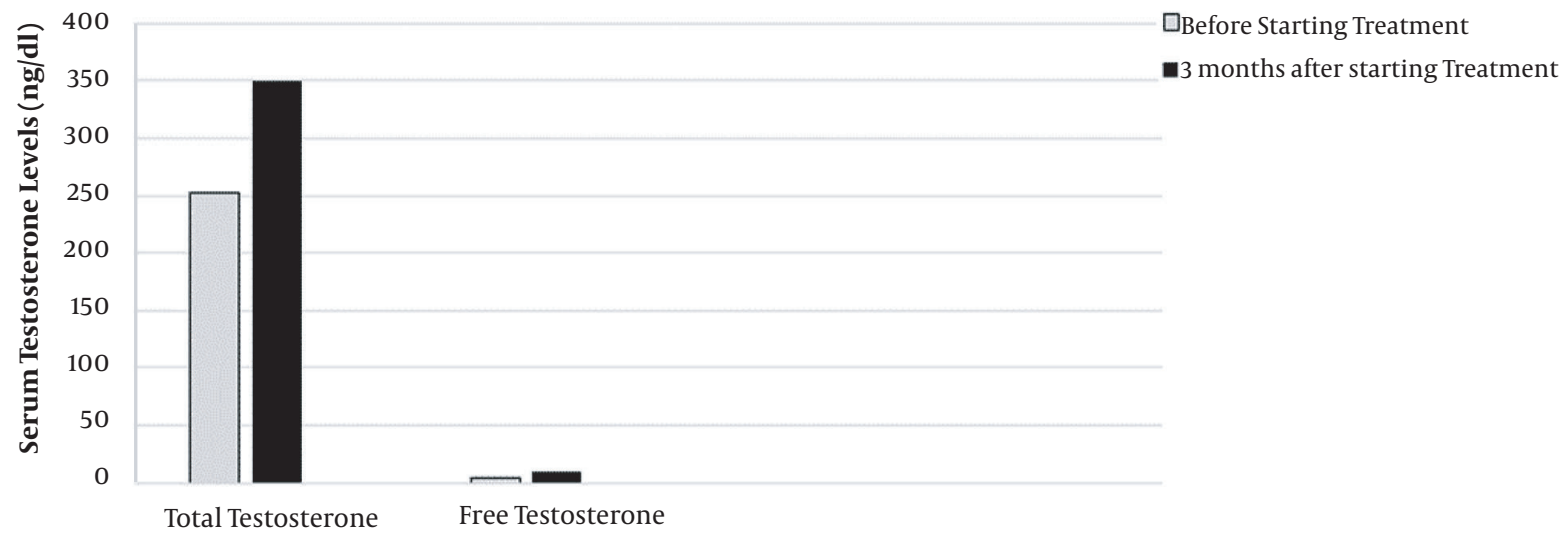

Figure 2. Testosterone levels before and 3 months after starting treatment 


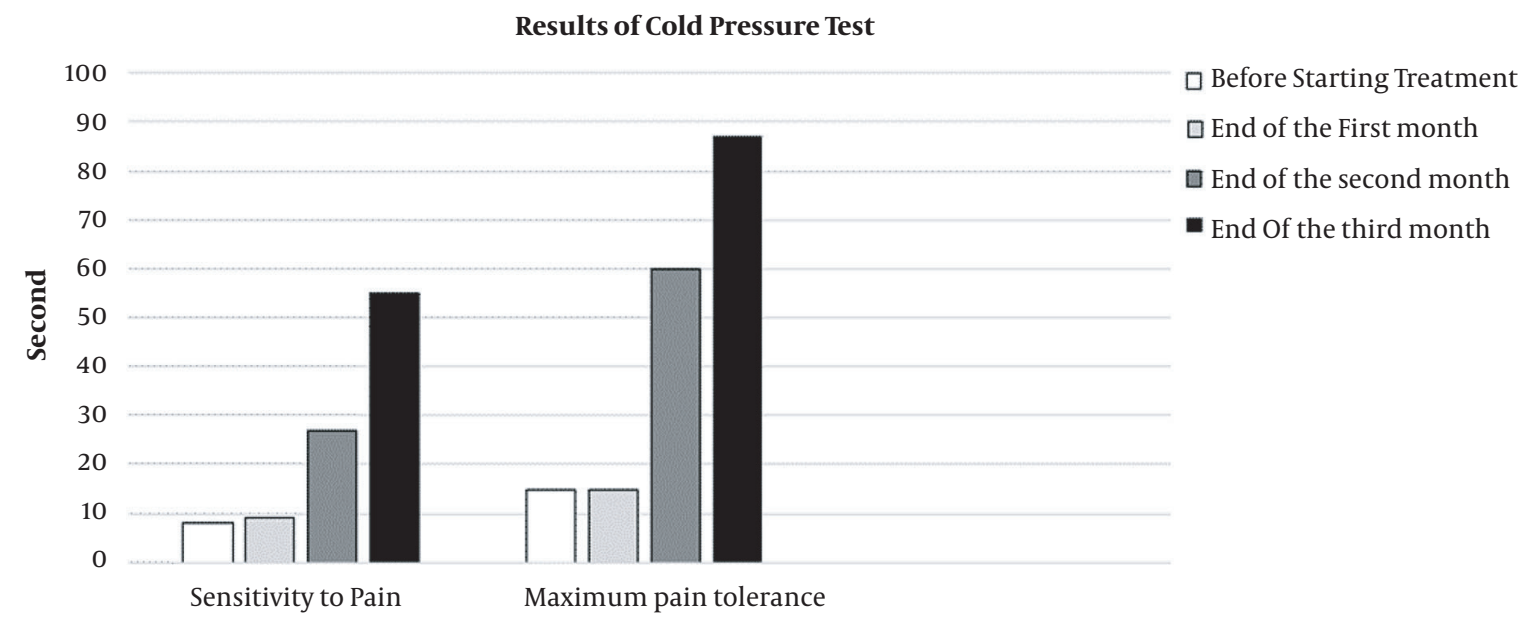

Figure 3. Results of cold pressure test

\section{Footnotes}

Authors' Contributions: Study concept and design: N.Ch. \& Sh. N; Data analysis and interpretation: N.Ch. \& M. M; Manuscript drafting: K. Gh. \& M. M; Critical revision of the manuscript for important intellectual content: N. Ch. \& K. Gh. \& M. M; Statistical analysis: N. Ch. \& Sh. N.

Conflict of Interests: The authors declared no conflict of interests.

Ethical Approval: The interventions performed in this study were part of routine treatments and therefore did not require a code of ethics.

Funding/Support: The authors received no funding or support for this study.

Informed Consent: In this study, no intervention was performed outside the patient's treatment process, and only the patient's condition was described and measured.

\section{References}

1. Bawor M, Bami H, Dennis BB, Plater C, Worster A, Varenbut M, et al. Testosterone suppression in opioid users: a systematic review and meta-analysis. Drug Alcohol Depend. 2015;149:1-9. doi: 10.1016/j.drugalcdep.2015.01.038. [PubMed: 25702934].

2. Huang G, Travison TG, Edwards RR, Basaria S. Effects of testosterone replacement on pain catastrophizing and sleep quality in men with opioid-induced androgen deficiency. Pain Medicine. 2017;18(6):1070-6. doi: 10.1093/pm/pnw159.

3. Banihani SA. Ginger and Testosterone. Biomolecules. 2018;8(4):119. doi: 10.3390/biom8040119. [PubMed: 30360442]. [PubMed Central: PMC6316093].

4. Basaria S, Travison TG, Alford D, Knapp PE, Teeter K, Cahalan C, et al. Effects of testosterone replacement in men with opioid-induced androgen deficiency: a randomized controlled trial. Pain. 2015;156(2):280-
8. doi: 10.1097/01.j.pain.0000460308.86819.aa. [PubMed: 25599449]. [PubMed Central: PMC6036339].

5. Cepeda MS, Zhu V, Vorsanger G, Eichenbaum G. Effect of Opioids on Testosterone Levels: Cross-Sectional Study using NHANES. Pain Med. 2015;16(12):2235-42. doi: 10.1111/pme.12843. [PubMed: 26177122].

6. Jasuja GK, Ameli O, Reisman JI, Rose AJ, Miller DR, Berlowitz DR, et al. Health Outcomes Among Long-term Opioid Users With Testosterone Prescription in the Veterans Health Administration.JAMA Netw Open. 2019;2(12). e1917141. doi: 10.1001/jamanetworkopen.2019.17141. [PubMed: 31825502]. [PubMed Central: PMC6991198].

7. Lee MO. A Comprehensive Review of Opioid-Induced Hyperalgesia. Pain Physician. 2011;3;14(2;3):145-61. doi: 10.36076/ppj.2011/14/145.

8. Kumar P, Kumar N, Thakur DS, Patidar A. Male hypogonadism: Symptoms and treatment. J Adv Pharm Technol Res. 2010;1(3):297-301. doi: 10.4103/0110-5558.72420. [PubMed: 22247861]. [PubMed Central: PMC3255409].

9. Winters SJ. Introduction to Endocrine Disorders in Men. A Case-Based Guide to Clinical Endocrinology. Springer; 2022. p. 261-2.

10. Kum E, Buckley N, de Leon-Casasola O, Lema M, Busse JW. Attitudes Towards and Management of Opioid-induced Hyperalgesia: A Survey of Chronic Pain Practitioners. Clin J Pain. 2020;36(5):359-64. doi: 10.1097/AJP.0000000000000814. [PubMed: 32028382].

11. Vargas-Schaffer G, Paquet S, Neron A, Cogan J. Opioid Induced Hyperalgesia, a Research Phenomenon or a Clinical Reality? Results of a Canadian Survey. J Pers Med. 2020;10(2). doi: 10.3390/jpm10020027. [PubMed: 32326188]. [PubMed Central: PMC7354508].

12. Belkin M, Reinheimer HS, Levy J, Johnson B. Ameliorative response to detoxification, psychotherapy, and medical management in patients maintained on opioids for pain. Am J Addict. 2017;26(7):738-43. doi: 10.1111/ajad.12605. [PubMed: 28800186].

13. Lentini M, Scalia J, Lebel FB, Touma F, Jhajj A, Darlington PJ, et al. Association Between Pain Catastrophizing and Pain and Cardiovascular Changes During a Cold-Pressor Test in Athletes. J Athl Train. 2021;56(5):473-83. doi: 10.4085/1062-6050-016-20. [PubMed: 34000018]. [PubMed Central: PMC8130774].

14. Peles E, Schreiber S, Gordon J, Adelson M. Significantly higher methadone dose for methadone maintenance treatment (MMT) patients with chronic pain. Pain. 2005;113(3):340-6. doi: 10.1016/j.pain.2004.11.011. [PubMed: 15661442]. 